\title{
Statistical Quark Model for the Nucleon Structure Function
}

\author{
Carlos Mirez*, Lauro Tomio*, Luis A. Trevisan ${ }^{\dagger}$ and Tobias Frederico** \\ ${ }^{*}$ Instituto de Fisica Teórica, Universidade Estadual Paulista, 01405-900, São Paulo, SP,Brazil. \\ †Departamento de Matemática e Estatística, Universidade Estadual de Ponta Grossa, 84010-790, \\ Ponta Grossa, PR, Brazil. \\ ${ }^{* *}$ Departamento de Física, Instituto Tecnológico de Aeronáutica, CTA, 12228-900, São José dos \\ Campos, SP, Brazil.
}

\begin{abstract}
A statistical quark model, with quark energy levels given by a central linear confining potential is used to obtain the light sea-quark asymmetry, $\bar{d} / \bar{u}$, and also for the ratio $d / u$, inside the nucleon. After adjusting a temperature parameter by the Gottfried sum rule violation, and chemical potentials by the valence up and down quark normalizations, the results are compared with experimental data available.
\end{abstract}

Keywords: flavor symmetries, structure function, quark models

PACS: $11.30 . \mathrm{Hv}, 12.39 . \mathrm{x}, 14.65 . \mathrm{Bt}$

We report results for the light sea-quark asymmetry, $\bar{d} / \bar{u}$ and also for the ratio $d / u$ obtained in a statistical quark model [1], where the quark energy levels are given by a central linear confining potential [2]. The model is parameterized by experimental data, with the Gottfried sum rule (GSR) violation [3] being adjusted by a temperature parameter, and with two chemical potentials to fix the flavor normalization inside the nucleon. From the analysis of the E866 experiments [4], the value for the violation of the GSR is $I_{G S R}=\int_{0}^{1}[\bar{d}(x)-\bar{u}(x)] d x=0.118 \pm 0.012$. The up and down sea quarks are assumed to be massless, with levels given by a linear scalar plus vector potential [2]. The statistical quark model is outlined in Ref. [5]. In this reference, the model was shown to give a good description of the strangeness content of the nucleon [5] when compared with experimental data.

In Fig. 1, we report the results we have obtained by the model for the ratios $\bar{d} / \bar{u}$ [in the left frame of Fig. 1] and $d / u$ [right frame of Fig.1], as functions of the Bjorken scale $x$. Initially, in spite of the fact that we fit the GSR violation with such a model, we obtain a constant ratio between the proton and neutron structure functions [constant dashed line of Fig.1]. Next, we incorporate in the model contributions from gluonic splitting processes, which is represented by the parameter $\alpha_{s}$. This is shown in the left frame of Fig. 1 by the small dashed curve, and in the right panel by the solid line. In the results shown for $\bar{d} / \bar{u}$, in the left panel of Fig.1, we also verify how the substructure of the constituent quarks can affect the results. Such substructure is obtained from a model for the structure function of a valence quark in the pion, given by the following parametrization [1]: $q_{\pi}(x)=N_{\pi} x^{a-1}(1+A \sqrt{x}+B x)(1-x)^{D}$, where: $N_{\pi} \equiv$ $1.212+0.498 s+0.009 s^{2}, A \equiv-0.037-0.578 s, a \equiv 0.517-0.020 s, B \equiv 0.241+0.251 s$, $D \equiv 0.383+0.624 s$, with $s \equiv \ln \left[\ln \left(Q^{2} / 0.204^{2}\right) / \ln \left(\mu^{2} / 0.204^{2}\right)\right]$ evaluated at $\mu^{2}=0.26$ $\mathrm{GeV}^{2}$ [6]. Using this prescription, the constituent structure of the quark (antiquark) in 
the nucleon is given by $q(x)=-\left.\int_{x}^{1} \frac{\partial}{\partial z} q_{\pi}\left(z, Q^{2}\right)\right|_{z=x / y} q_{\text {model }}(y) d y$, where $q_{\text {model }}(y)$ is the quark structure function given by the statistical model. In Fig.1, the experimental data are from Refs. [7, 8]; in the right panel we also show on- and off-shell results given in Ref. [9].
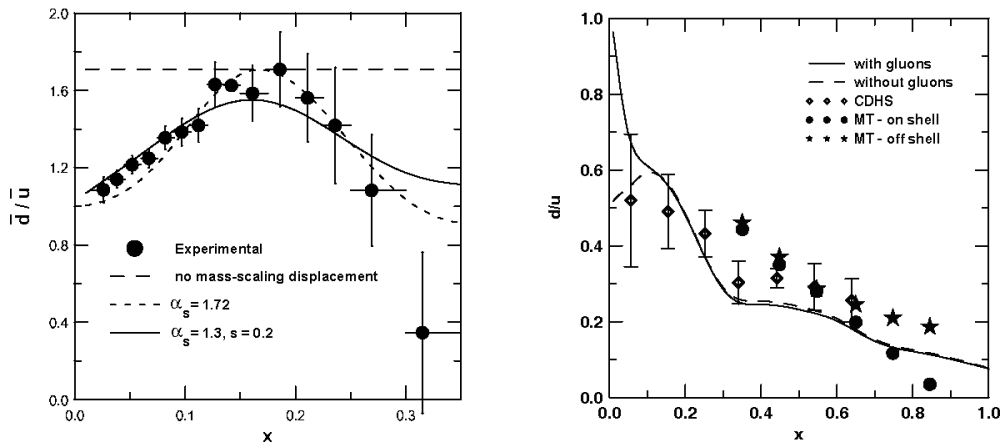

FIGURE 1. Lefi panel: Model results for $\bar{d} / \bar{u}$, as a function of $x$, compared with data [7]. Without mass-scaling displacement, we have the constant (long-dashed line) results. With the small-dashed-line and solid-line curves, we consider gluonic contributions, without quark substructure (small-dashed-line, for $\alpha_{s}=1.72$ ); and with quark substructure (solid-line, with $\alpha_{s}=1.3$ and $s=0.2$ ). Right panel: $d / u$ distribution inside the proton as a function of $x$. The results, considering the mass-scaling displacement, without gluon splitting (dashed line) and with gluon splitting (solid line), are compared with experimental data [8]. We also show on-shell (solid circles) and off-shell (stars) calculations of Ref. [9].

Finally, with the statistical model parametrization reproducing the $\Delta$ resonance, we should note that instanton induced interactions between quarks [10] are responsible for the mass shift between nucleon and $\Delta$. The qualitative difference between the structure functions comes from the fact that light quarks with different flavors ( $u$ and $d$ and corresponding antiparticles) also have different current masses. So, a simple mathematical trick, based in the Dirac's delta distribution, was used in Ref. [1] to obtain the shift of the given structure functions (which are equal a priori). At the end, after considering the contributions from gluon splitting processes [11], a quite good fit is obtained for the ratios $d(x) / u(x)$ and $\bar{d}(x) / \bar{u}(x)$ in the proton.

\section{ACKNOWLEDGMENTS}

We thank Brazilian agencies FAPESP and CNPq for partial support.

\section{REFERENCES}

1. L.A. Trevisan, C. Mirez, T. Frederico and L. Tomio Eur. Phys. J. C 56, 221 (2008), Nucl. Phys. A 790 $522(2007)$.

2. P.L. Ferreira, J.A. Helayel, and N. Zagury, N. Cim. A 55, 215 (1980).

3. K. Gottfried, Phys. Rev. Lett. 18, 1174 (1967).

4. R.S. Towell et al., Phys. Rev. D 64,052002 (2002).

5. L.A. Trevisan, T. Frederico and L. Tomio, Eur. Phys. J. C11, 351 (1999).

6. M. Glück, E. Reya, and I. Schienbein, Eur. Phys. J. C 10, 313 (1999).

7. J.C. Peng et al., Phys. Rev. D 58, 092004 (1998). E.A. Hawker et al., Phys. Rev. Lett. 80, 3715 (1998).

8. H. Abramowicz et al., Z. Phys. C 25, 29 (1983).

9. W. Melnitchouk, A.W. Thomas, Phys. Lett. B 377, 11 (1996).

10. A.E. Dorokhov, N.I. Kochelev and Yu.A. Zubov, Sov. J. of Part. Nucl. 23, 522 (1992).

11. G. Altarelli and G. Parisi, Nucl. Phys. B 126, 298 (1977). 
Copyright of AIP Conference Proceedings is the property of American Institute of Physics and its content may not be copied or emailed to multiple sites or posted to a listserv without the copyright holder's express written permission. However, users may print, download, or email articles for individual use. 